\title{
Delegated portfolio management under ambiguity aversion
}

\author{
Annalisa Fabretti ${ }^{\mathrm{a}}$, Stefano Herzel ${ }^{\mathrm{a}}$, Mustafa Ç. Pınar ${ }^{\mathrm{b}, *}$ \\ a Department of Economics and Finance, University of Rome “Tor Vergata”, Via Columbia 2, Rome, Italy \\ ${ }^{\mathrm{b}}$ Department of Industrial Engineering, Bilkent University, 06800 Bilkent, Ankara, Turkey
}

\section{A R T I C L E I N F O}

\section{Article history:}

Received 3 January 2013

Received in revised form

5 February 2014

Accepted 5 February 2014

Available online 15 February 2014

\section{Keywords:}

Delegated portfolio management

Ambiguity

Robust optimization

\begin{abstract}
A B S T R A C T
We examine the problem of setting optimal incentives for a portfolio manager hired by an investor who wants to induce ambiguity-robust portfolio choices with respect to estimation errors in expected returns. Adopting a worst-case max-min approach we obtain the optimal compensation in various cases where the investor and the manager, adopt or relinquish an ambiguity averse attitude. We also provide examples of applications to real market data.
\end{abstract}

(c) 2014 Elsevier B.V. All rights reserved.

\section{Introduction}

The purpose of this paper is to provide a model for the study of a problem of delegated portfolio management when there is uncertainty on the estimates of the expected returns of the assets in the investment set and the agents are averse to such an ambiguity.

We are interested in analyzing the effects of ambiguity aversion in portfolio choices and managerial fees. To this end we assume that a portfolio manager with an exponential utility function is hired by an investor who pays him a fee on the final wealth produced by the selected portfolio strategy. The manager accepts the contract under the condition that his compensation is at least as large as a minimum level that he sets based on his minimum acceptable level of utility.

This kind of problem is studied within the theory of Delegated Portfolio Management where the optimal form of a contract between investors and managers is investigated. The seminal paper of this theory is due to Bhattacharya and Pfleiderer (1985) [7]. A nice review of the main contributions can be found in Stracca (2006) [22]. For more recent references see also Fabretti and Herzel (2012) [15]. Our approach integrates an important line of research in robust portfolio selection; see e.g., $[2,4,9,10,18,14,13,17,19,23]$ and references therein. Instead of assuming a probability distribution and formulating a stochastic optimization problem, Robust Optimization (RO) confines data uncertainties into an uncertainty set, and follows a worst-case approach which takes full responsibility for all occurrences of data within the uncertainty set, an

\footnotetext{
* Corresponding author. Tel.: +90 3122902603.

E-mail addresses: annalisa.fabretti@uniroma2.it (A. Fabretti), stefano.herzel@uniroma2.it (S. Herzel), mustafap@bilkent.edu.tr (M.Ç. Pınar).
}

approach akin to the min-max approach of robust control. Suitable uncertainty sets (ellipsoidal, polyhedral) are justified according to the problem context, and RO methodology proceeds by transforming the min-max problem into a "tractable" (that can be processed by available efficient algorithms) optimization problem. In the context of portfolio selection, it is well-known that distribution of expected returns is not known precisely, and that portfolio composition is particularly sensitive to expected return data $[5,6,8,12]$. Several authors in the references [3,4,9-11,14,13,17,19,21,23] addressed this problem by applying robust optimization techniques to variants of the portfolio selection problems.

In general, past contributions on robust portfolio selection, with the exception of Garlappi et al. (2007) [17], rely on numerical solution of optimization problems, whereas in this paper we obtain a closed-form robust portfolio selection rule. We consider a one period economy with $n$ risky assets following a multivariate return distribution and a risk-less asset. In this world there is an investor who is averse to estimation error in the expected return estimates of the risky assets (we call this ambiguity aversion). The investor, unable (or unwilling) to undertake the investment directly by herself, wants to hire a portfolio manager. She offers a contract which is a linear function of the final (random) wealth, and faces the problem of selecting a suitable fee to be paid to the manager (by maximizing ambiguity robust expected final wealth after paying off the manager), which should be sufficiently high to attract the manager. The manager accepts the contract provided that his utility reservation constraint is satisfied. The main objective of our study is to formulate a model that is simple enough to get explicit results but also sufficiently structured to address important issues such as the impact of the investor's ambiguity aversion. This impact is measurable from the explicit formulae we obtain. 
We will show an application of our results to an example with real market data, determining the sharing rules for different cases of investment strategies with a constraint based on socially responsible rules. This example is interesting because we can compare two opposite effects due to the restriction of the investment set, the first one negative, because of the ruled out investment opportunities, the second one positive, because more socially responsible firms may be, according to some professionals and academics [1], more "transparent" and hence more desirable for an ambiguity averse decision maker. For details, the reader is referred to the extended version of our paper [16].

\section{Portfolio strategies under ambiguity aversion}

Here we consider the problem of an Ambiguity Averse (AA) agent with a Constant Absolute Risk Averse (CARA) utility with risk aversion $\alpha$, e.g. a negative exponential utility function. The agent maximizes his utility trading in one risk-less asset with return $R \geq$ 1 and in $n$ risky assets with return vector $\mathbf{X}$ which follows a Gaussian law with mean $\overline{\mathbf{X}}$ and positive definite variance-covariance matrix $\Sigma$.

We model ambiguity as uncertainty in the mean of the return vector $\mathbf{X}$. We assume that an AA agent is uncertain about the true mean $\overline{\mathbf{X}}$, but assumes that it belongs to the set

$U_{\hat{X}}^{(\epsilon)}=\left\{\mathbf{Y} \in \mathbb{R}^{n} \mid(\mathbf{Y}-\hat{\mathbf{X}})^{T} \Sigma^{-1}(\mathbf{Y}-\hat{\mathbf{X}}) \leq \epsilon^{2}\right\}$,

that is an $n$-dimensional ellipsoid centered at $\hat{\mathbf{X}}$ (the estimated mean) with radius $\epsilon$, which we call the "level of ambiguity aversion". The idea is that the decisions of an AA agent are taken by considering the worst case occurrences of the true mean $\overline{\mathbf{X}}$ within the set $U_{\hat{X}}^{(\epsilon)}$. Therefore, more conservative choices are taken when the set is larger, i.e. for greater values of $\epsilon$, while an "Ambiguity Neutral" agent who does not have any doubt about errors in the estimated values sets $\epsilon$ equal to zero and assumes that the estimated mean $\hat{\mathbf{X}}$ is equal to the true mean $\overline{\mathbf{X}}$. The differences between the true mean $\overline{\mathbf{X}}$ and its forecast $\hat{\mathbf{X}}$ depend on the variance of the returns, hence they are scaled by the inverse of the covariance matrix. The random variable $(\overline{\mathbf{X}}-\hat{\mathbf{X}})^{T} \Sigma^{-1}(\overline{\mathbf{X}}-\hat{\mathbf{X}})$ has a well known distribution (under standard assumptions on the returns), and this fact, as we will see in Section 5, can be exploited to set the values for $\epsilon$ in order to get the desired likelihood for the true mean to belong to the set $U_{\hat{X}}^{(\epsilon)}$.

Let us define the vector of estimated excess expected returns $\hat{\mu}=\hat{\mathbf{X}}-R \mathbf{1}$ where $\mathbf{1}$ is a $n$-vector of ones and the quantity $\hat{H}=\sqrt{\hat{\mu}^{T} \Sigma^{-1} \hat{\mu}}$. It is well known that $\hat{H}$ is the maximal Sharpe Ratio obtainable in a market model with parameters $\Sigma$ and $\hat{\mu}$. Note that it depends on the estimated value of the expected excess return which may be different from the unknown real value. An AA investor who believes that the real expected return $\overline{\mathbf{X}}$ belongs to the ellipsoid $U_{\hat{X}}^{(\epsilon)}$ solves the problem

$\max _{\omega} \min _{\overline{\mathbf{X}} \in U_{\hat{X}}^{(\epsilon)}} \frac{(\overline{\mathbf{X}}-R \mathbf{1})^{T} \omega}{\sqrt{\omega^{T} \Sigma \omega}}$

where $\omega$ is the $n$-dimensional portfolio vector (i.e. $\omega_{i}$ represents the money invested in the ith risky asset). After solving the inner problem, the optimization becomes

$$
\max _{\omega} \frac{(\hat{\mathbf{X}}-R \mathbf{1})^{T} \omega-\epsilon \sqrt{\omega^{T} \Sigma \omega}}{\sqrt{\omega^{T} \Sigma \omega}}=\hat{H}-\epsilon .
$$

Hence we can define the "ambiguity averse" Sharpe Ratio, with level of ambiguity aversion $\epsilon$, as $\hat{H}_{\epsilon}=\hat{H}-\epsilon$. Therefore $\hat{H}_{\epsilon}$ is the maximal Sharpe Ratio according to an AA investor. Note that it is always smaller than the Sharpe Ratio $\hat{H}$ based on the estimated values and that it may also be negative. A negative $\hat{H}_{\epsilon}$ means that the $\mathrm{AA}$ investor will refrain from investing in the risky assets.

Let $W_{0}$ be the initial wealth to be invested, then the AA agent with CARA utility with risk-aversion $\alpha$ selects a portfolio $\omega$ by solving the problem

$\max _{\omega} \min _{\overline{\mathbf{X}} \in U_{\hat{\mathbf{X}}}^{(\epsilon)}} \mathbb{E}\left[-e^{-\alpha\left(\omega^{T}(\mathbf{X}-R \mathbf{1})+W_{0} R\right)}\right]$

where $\mathbf{X} \sim N(\overline{\mathbf{X}}, \Sigma)$. The following proposition shows that (3) has a closed form solution.

Proposition 1. Let returns $\mathbf{X}$ be normally distributed with mean $\overline{\mathbf{X}}$ and variance $\Sigma$. An AA agent with a CARA utility with risk-aversion $\alpha$ and ambiguity set $U_{\hat{X}}^{(\epsilon)}$ selects the same portfolio strategy as an agent who is ambiguity neutral but is risk-averse with a coefficient of riskaversion $\hat{\alpha}_{\epsilon}=\alpha \frac{\hat{H}}{\hat{H}_{\epsilon}}$, provided that $\hat{H}_{\epsilon}>0$. When $\hat{H}_{\epsilon} \leq 0$ the $A A$ agent is equivalent to an agent with an infinite risk-aversion.

Proof. An agent who is neutral with respect to ambiguity assumes that the true mean $\overline{\mathbf{X}}$ is equal to the estimated mean $\hat{\mathbf{X}}$ and selects the optimal strategy solving the problem

$$
\max _{\omega} \mathbb{E}\left[-e^{-\alpha\left(\omega^{T}(\mathbf{X}-R \mathbf{1})+W_{0} R\right)}\right],
$$

where $\mathbf{X} \sim N(\overline{\mathbf{X}}, \Sigma)$. The solution of $(4)$ is

$\bar{\omega}=\frac{1}{\alpha} \Sigma^{-1}(\overline{\mathbf{X}}-R \mathbf{1})=\frac{1}{\alpha} \Sigma^{-1} \hat{\mu}$,

where the last equality follows from the assumption of neutrality with respect to ambiguity.

We prove that the optimal portfolio strategy for problem (3) is

$\hat{\omega}= \begin{cases}\frac{1}{\alpha}\left(\frac{\hat{H}_{\epsilon}}{\hat{H}}\right) \Sigma^{-1} \hat{\mu} & \text { if } \hat{H}_{\epsilon}>0 \\ 0 & \text { if } \hat{H}_{\epsilon} \leq 0 .\end{cases}$

Then the result follows immediately from comparing the two solutions (5) and (6).

To obtain (6) we start by computing the expectation in (3) and reformulating the problem as

$\max _{\omega} \min _{\overline{\mathbf{x}} \in U_{\hat{X}}^{(\epsilon)}}-\exp \left(-\alpha \overline{\mathbf{X}}-R \mathbf{1}^{T} \omega+\frac{\alpha^{2}}{2} \omega^{T} \Sigma \omega-\alpha W_{0} R\right)$.

Taking the logarithm and solving the inner minimization problem (see Garlappi et al. [17]) we get

$\max _{\omega} \hat{\mu}^{T} \omega-\frac{\alpha}{2} \omega^{T} \Sigma \omega-\epsilon \sqrt{\omega^{T} \Sigma \omega}$.

Under the hypothesis that the problem admits a solution $\hat{\omega}$ different from zero, first-order conditions (note that the objective is a concave function of $\omega$ )

$\hat{\mu}-\alpha \Sigma \omega-\epsilon \frac{\Sigma \omega}{\sqrt{\omega^{T} \Sigma \omega}}=0$,

are both necessary and sufficient. Straightforward computations then lead to (6).

The previous result states that increasing the level of ambiguity aversion leads to an increase in the risk aversion. More precisely, the parameter of risk aversion increases by a factor that is proportional to the ratio between the estimated Sharpe ratio $\hat{H}$ and the AA Sharpe ratio $\hat{H}_{\epsilon}$. When the uncertainty on the estimate is so high that $\hat{H}_{\epsilon}$ is not positive, the agent does not assume any position in the risky assets and put all his wealth into the risk-free investment, i.e. he behaves as if his risk aversion is infinite. 


\section{Delegated portfolio management under ambiguity aversion}

Now we consider the problem of an investor who wishes to delegate the management of her wealth to a portfolio manager. We assume that the investor is risk-neutral, while the portfolio manager has a CARA utility with risk-aversion $\alpha$. Both the investor and the manager may also be averse to ambiguity.

The investor allocates a capital $W_{0}$ to the portfolio manager with the mandate to form a portfolio with the assets available in the market, including the risk free asset. At the end of the period, the investor will compensate the manager with a fraction $b$ of the final value of the portfolio, keeping the rest for herself. Hence an Ambiguity Neutral manager with risk aversion coefficient $\alpha$ determines the optimal portfolio allocation by solving

$\max _{\omega} \mathbb{E}\left[-e^{-\alpha b\left(\omega^{T}(\mathbf{X}-R \mathbf{1})+W_{0} R\right)}\right]$,

where $\mathbf{X} \sim N(\hat{\mathbf{X}}, \Sigma)$. We also assume that the manager sets a minimum level $b_{0}$ for the fraction of the final value of the portfolio to accept the contract and a maximum level that is equal to 1 . The choice of $b_{0}$ will be discussed in detail in Section 4 . Therefore, the problem of the investor is to choose the best value for the compensation factor $b$ according to her utility, by keeping in mind that the value of $b$ also affects the portfolio strategy of the manager.

From Proposition 1 it follows that an AA manager is equivalent, in terms of portfolio choices, to an Ambiguity Neutral manager with an adjusted risk-aversion. For this reason, we can consider, as a general case, the problem of an AA investor who hires an Ambiguity Neutral manager with risk aversion $\alpha$, that is

$\max _{b_{0} \leq b \leq 1} \min _{\overline{\mathbf{X}} \in U_{\hat{\mathbf{X}}}^{(\delta)}} \mathbb{E}\left[(1-b)\left(\hat{\omega}^{T}(\mathbf{X}-R \mathbf{1})+W_{0} R\right)\right]$

where $\mathbf{X} \sim N(\overline{\mathbf{X}}, \Sigma)$ and the allocation $\hat{\omega}$ is chosen by the manager solving (7), that is

$\hat{\omega}=\frac{1}{\alpha b} \Sigma^{-1} \hat{\mu}$.

Proposition 2. The optimal compensation factor for an AA investor with ambiguity set $U_{\hat{\mathrm{X}}}^{(\delta)}$ dealing with a manager with CARA utility with risk-aversion $\alpha$ is

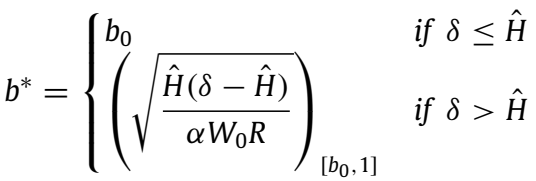

where $(\cdot)_{\left[b_{0}, 1\right]}$ represents projection onto the interval $\left[b_{0}, 1\right]$.

Proof. We consider first the minimum in (8), that is a convex optimization problem satisfying Slater condition, therefore optimality conditions are both necessary and sufficient. The Lagrangian function is $\mathcal{L}(\overline{\mathbf{X}}, \lambda)=\hat{\omega}^{T}(\overline{\mathbf{X}}-\mathbf{1} R)+W_{0} R-\lambda\left(\delta^{2}-(\overline{\mathbf{X}}-\hat{\mathbf{X}})^{T} \Sigma^{-1}(\overline{\mathbf{X}}-\hat{\mathbf{X}})\right)$, and first order conditions with respect to $\overline{\mathbf{X}}$ admit the solution $\overline{\mathbf{X}}^{*}=$ $\hat{\mathbf{X}}-\frac{1}{2 \lambda} \Sigma \hat{\omega}$ which gives $\mathcal{L}\left(\overline{\mathbf{X}}^{*}, \lambda\right)=\hat{\omega}^{T} \hat{\mu}+W_{0} R-\frac{1}{4 \lambda} \hat{\omega}^{T} \Sigma \hat{\omega}-\lambda \delta^{2}$. Differentiating and solving for $\lambda$ gives $\lambda^{*}=\frac{\sqrt{\hat{\omega}^{T} \Sigma \hat{\omega}}}{2 \delta}$, which transforms the problem (8) into

$\max _{b}(1-b)\left(\hat{\omega}^{T} \hat{\mu}+W_{0} R-\delta \sqrt{\hat{\omega}^{T} \Sigma \hat{\omega}}\right)$.

Substituting the optimal allocation $\hat{\omega}$ given by (9) we obtain $\max _{b}$ $(1-b)\left(W_{0} R+\frac{\hat{H}(\hat{H}-\delta)}{\alpha b}\right)$. The objective function is convex for $\hat{H} \geq$ $\delta$ and concave for $\hat{H}<\delta$. Its first derivative is $-W_{0} R-\frac{\hat{H}(\hat{H}-\delta)}{\alpha b^{2}}$ which is always negative if $\hat{H} \geq \delta$, while it admits the root $\sqrt{\frac{\hat{H}(\delta-\hat{H})}{\alpha W_{0} R}}$ if $\delta>\hat{H}$.
Proposition 2 shows that if $\delta \leq \hat{H}$, the investor assigns to the manager the minimum possible share that is sufficient to hire him, because a smaller share keeps the manager greedy and ready to assume riskier positions, which is adequate for the risk-neutral investor. If $\delta>\hat{H}$ the investor is so pessimistic about the quality of the mean return estimates that she would rather stay away from the risky assets. In this case increasing $b$ has, for the investor, both positive and negative effects. Positive because it induces the manager to be more risk-averse, negative because it decreases the investor's wealth. The value of $b^{*}$ for the case $\delta>\hat{H}$ finds the balance between these two effects. The projection onto the interval $\left[b_{0}, 1\right]$ is due to the constraint imposed to the original problem.

We remark that, as shown by Proposition 1, when the ambiguity aversion $\delta$ is greater than $\hat{H}$, the optimal choice for a risk-neutral (and also for a risk-averse) investor would be to refrain from investing into risky assets.

The case of an AA portfolio manager is obtained as a corollary of the previous result. The notation $\hat{H}_{\delta}$ is defined analogously to $\hat{H}_{\epsilon}$.

Corollary 1. The optimal compensation factor for an AA investor with ambiguity set $U_{\hat{X}}^{(\delta)}$ dealing with a CARA utility manager with riskaversion $\alpha$ and ambiguity set $U_{\hat{X}}^{(\epsilon)}$ is

$b^{*}= \begin{cases}\left(\sqrt{\left.\frac{\left(\hat{H}_{\epsilon}\right)\left(-\hat{H}_{\delta}\right)}{\alpha W_{0} R}\right)_{\left[b_{0}, 1\right]}}\right. & \text { if } \hat{H}_{\epsilon}>0 \text { and } \hat{H}_{\delta}<0 \\ b_{0} & \text { o.w. }\end{cases}$

Proof. It is sufficient to use Proposition 1 and substitute $\hat{\alpha}_{\epsilon}$ for $\alpha$ into (10).

When the manager is strongly diffident about the estimates (i.e. his AA Sharpe ratio is negative), he will invest all the wealth in the risk-free asset, independently from the incentives received by the investor, therefore the best strategy for the investor is to set the compensation to the minimum level $b_{0}$ set by the participation constraint. Indeed, the quantity $b_{0}$ plays a key role in this problem and in the next section we will see how it can be determined.

To focus our analysis on the effects of ambiguity aversion we assumed that the investor is risk-neutral. In the case of a risk averse investor all formulas become more complicated, but it is still possible to show that the optimal sharing factor is increasing with the risk aversion of the investor and decreasing with the risk aversion of the manager. This is due to the fact that increasing the sharing factor increases the manager's risk aversion and therefore the investor uses it to align the manager's attitude towards risk with her own.

\section{Setting the participation constraint}

We assumed that the AA manager accepts the contract only if the investor offers a share of the final wealth greater than a minimum level $b_{0}$ chosen by the manager. The choice of $b_{0}$ depends on a minimum level of utility $\bar{U}$ that the manager expects to receive after signing the contract. The relation between $b_{0}$ and $\bar{U}$ is given by

$$
\max _{\omega} \min _{\overline{\mathbf{X}} \in U_{\hat{X}}^{(\epsilon)}} E\left[-e^{-\alpha b_{0}\left(\omega^{T}(\mathbf{X}-R \mathbf{1})+W_{0} R\right)}\right]=\bar{U},
$$

where $\mathbf{X} \sim N(\overline{\mathbf{X}}, \Sigma)$. We know that the optimal strategy selected by the manager is $\hat{\omega}=\mathbb{1}_{\hat{H}_{\epsilon}>0}\left(\frac{\hat{H}_{\epsilon}}{\alpha b_{0} \hat{H}}\right) \Sigma^{-1} \hat{\mu}$, where $\mathbb{1}_{A}$ is the characteristic function of the set $A$, and that (11) is equivalent to $-\alpha b_{0}$ $\left(\hat{\omega}^{T} \hat{\mu}+W_{0} R-\epsilon \sqrt{\hat{\omega}^{T} \Sigma \hat{\omega}}\right)+\frac{\left(\alpha b_{0}\right)^{2}}{2} \hat{\omega}^{T} \Sigma \hat{\omega}=\log (-\bar{U})$. After some substitutions we obtain

$b_{0}=\frac{1}{\alpha W_{0} R}\left(-\log (-\bar{U})-\frac{1}{2} \hat{H}_{\epsilon}^{2} \mathbb{1}_{\hat{H}_{\epsilon}>0}\right)$. 
This formula shows that the minimal sharing factor $b_{0}$ increases with the required level of utility $\bar{U}$ and decreases with the Ambiguity Adjusted Sharpe Ratio $\hat{H}_{\epsilon}$. When $\hat{H}_{\epsilon}$ is positive, the portfolio manager is willing to invest in risky assets, and hence he reduces the sharing factor required to achieve the level of utility $\bar{U}$.

To determine $\bar{U}$ the manager should compute the utility that he may obtain from other competing job offers. As a general case we consider a manager who has the opportunity to work for another investor who offers the same amount of money $W_{0}$ to be invested in a set of assets, we denote by $\rho$ the level of ambiguity aversion of the manager and by $\bar{H}_{\rho}$ the ambiguity adjusted Sharpe Ratio on the alternative investment set. Assuming that the alternative contract provides a sharing factor $\bar{b}_{0}$, we can easily compute the utility achieved by the manager on the alternative contract, that is the reservation utility $\bar{U}=-e^{-\alpha \bar{b}_{0} W_{0} R-\frac{\bar{H}_{\rho}^{2}}{2}} \mathbb{1}_{\bar{H}_{\rho}>0}$. Substituting into (12) we determine the equation of the sharing factors

$b_{0}=\bar{b}_{0}+\frac{1}{2 \alpha W_{0} R}\left[\bar{H}_{\rho}^{2} \mathbb{1}_{\bar{H}_{\rho}>0}-\hat{H}_{\epsilon}^{2} \mathbb{1}_{\hat{H}_{\epsilon}>0}\right]$.

The sharing factor $b_{0}$ increases with the Ambiguity Adjusted slope of the capital market line in the alternative investment set, and decreases with the Ambiguity Adjusted slope of the capital market line in the actual investment set. Therefore there may be cases where, because of the relative difference in the levels of ambiguity, the manager is willing to decrease the level of the sharing factor. An interesting special case is when the alternative investment set has the same Sharpe ratio as the proposed one but without uncertainty on the estimates of the expected returns (i.e. $\bar{H}_{\rho}=\hat{H}$ ). In such a case the difference between $b_{0}$ and $\bar{b}_{0}$ may be interpreted as a "premium for ambiguity", as it represents the extra compensation required by the manager when two investment sets differ only by the uncertainty on the expected returns.

\section{An application}

As an example of application of our results to real market data, we consider an investment mandate subject to some restrictions imposed by the investor. In particular, we study investment restrictions due to principles related to Social Responsibility (and to its contrary). We want to analyze how the ambiguity aversions of an investor and of a portfolio manager affect their optimal sharing factor. As a proxy for the choice of a socially responsible investment strategy we chose the FTSE KLD Social Index (henceforth KLD index).

We choose the Vice Fund as a proxy for "non-sociallyresponsible" investments. The Vice Fund invests in companies engaged in the aerospace and defense industries, owners and operators, gaming facilities as well as manufacturers of gaming equipment, manufactures of tobacco products and producers of alcoholic beverages. We remark that there is no intersection between the investment sets considered by the two strategies. The proxy for the risk free assets is the one-month Treasury bill. We are equipped with three time series spanning the period from September 2002 to September 2012.

For each month $t$ we computed the sample mean $\hat{X}_{t}$ and variance $\Sigma_{t}$ of the monthly excess returns of the two indices on a moving window consisting of the previous $T=36$ months. From these quantities, applying formula (2), we computed the ex-ante optimal Sharpe ratios of three investment strategies: the first one, called the "conventional" strategy, that combines the risk-free asset with the two indexes, the second one, called the "green" strategy, which excludes the Vice index and the "black" strategy, which excludes the KLD index.

Fig. 1 represents the optimal Sharpe ratios of the three strategies. As expected, because of the benefits of diversification, the Sharpe ratio of the conventional strategy is always above the other

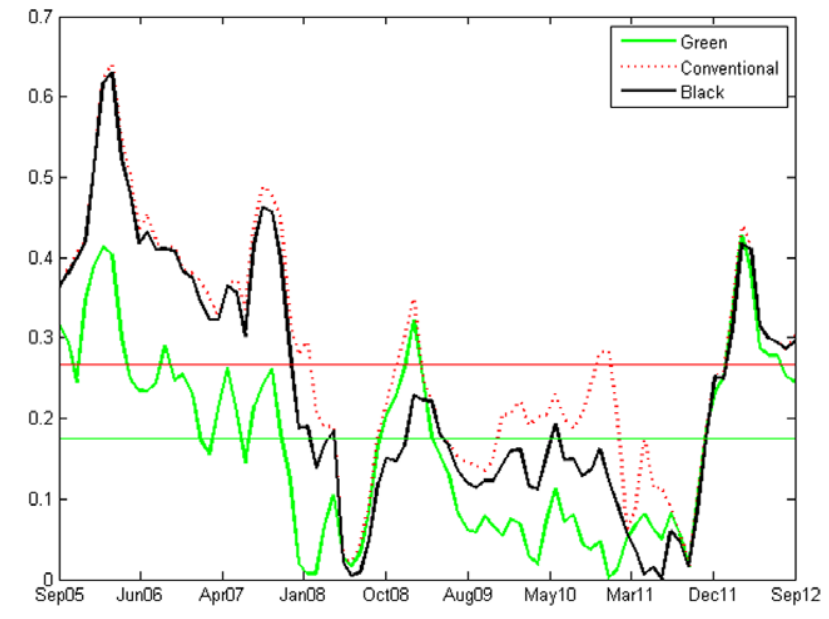

Fig. 1. Expected Sharpe ratios for the "conventional", the "black" and the "green" strategies. The straight lines represent the tolerances to ambiguity for $n=2$ (the conventional case) and $n=1$ (the other cases) at a probability level $p=0.7$. When the Sharpe ratio is below the corresponding tolerance line, an ambiguity averse investor should invest only in the risk-free asset.

two. For most of the period considered, the Sharpe ratio of the black strategy is higher than that of the green one. The only two subperiods when the Sharpe ratio of the green strategy was higher were between the end of 2008 and the beginning of 2009, and between March and October 2011. Note also that the only period where the "conventional" strategy significantly outperformed both the black and the green strategy is from August 2009 to June 2011.

To address the issue of ambiguity in the estimates of the expected returns on $n$ assets with a sample of length $T$, we use a well known result due to Hotelling (see Johnson and Wichern (1997) [20], page 212), which states that under standard assumptions on the time series of the returns, when $\Sigma$ is the sample covariance matrix, $\overline{\mathbf{X}}$ the exact mean and $\hat{\mathbf{X}}$ the sample mean, the random variable

$Z=\frac{T(T-n)}{(T-1) n}(\overline{\mathbf{X}}-\hat{\mathbf{X}})^{T} \Sigma^{-1}(\overline{\mathbf{X}}-\hat{\mathbf{X}})$

has an $F$-distribution with $n$ and $T-n$ degrees of freedom. Therefore, the level of ambiguity aversion $\epsilon$ can be obtained as a quantile of $Z$ corresponding to a given probability level $p$ of a sample mean falling inside the ellipsoid. The higher the aversion to ambiguity, the higher the value of $p$. In our example, we set $p=70 \%$, obtaining the values $\epsilon_{2}=0.267$ for $n=2$ and $\epsilon_{1}=0.175$ for $n=1$. The two constant lines in Fig. 1 correspond to these values. Note that the green and the black strategies have the same level of ambiguity $\epsilon$, because such a quantity only depends on $p$ and $n$. We remark that the value of $\epsilon$ is subjective as it depends on the level $p$ of confidence of the agent in the statistical estimates (and on the parameters $n$ and $T$ ). According to some studies (see e.g. [1] and the references therein), estimates on Socially Responsible stocks are more trustworthy, because Social Responsibility involves a more transparent management. An agent who adopts this view may assign lower values to the level of ambiguity aversion of Socially Responsible stocks. Here we take a neutral point of view setting the level of ambiguity aversion determined by the distribution of the random variable $Z$.

The analysis of Section 3 determines the optimal sharing factor $b$ as a function of market conditions, risk and ambiguity aversions, total wealth and the minimum acceptable fee. To study the effects of the investor's aversion to ambiguity on the compensation, we consider a conventional investment strategy with a portfolio manager who is neutral towards ambiguity. To determine a reference level for the factor $\alpha W_{0}$ appearing in (10) we assume that the manager would choose a portfolio composed only by the risky assets if 


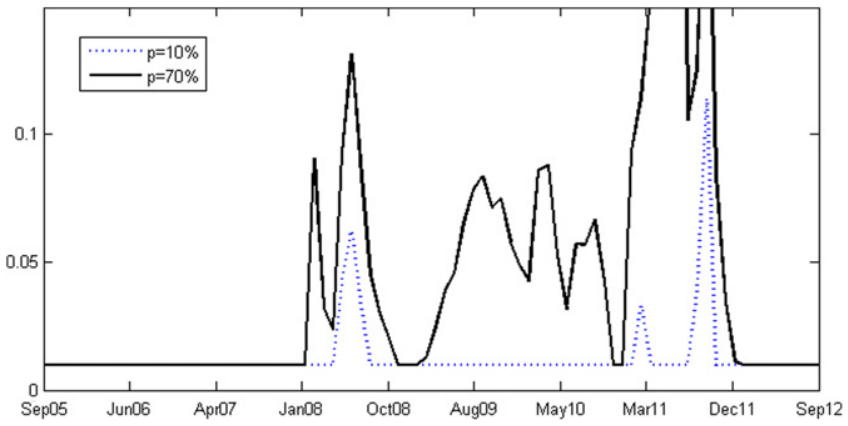

Fig. 2. Optimal sharing factor $b^{*}$ for two levels of ambiguity aversion. The initial wealth $W_{0}$ and manager's risk aversion $\alpha$ are assumed to satisfy (15). The minimum sharing factor is set to $b_{0}=1 \%$.

he had to decide where to invest $W_{0}$ on his own account. This assumption implies that $\mathbf{1}^{T} \hat{\omega}=W_{0}$, therefore, using formula (5) for the optimal portfolio $\hat{\omega}$, we get

$\alpha W_{0}=\mathbf{1}^{T} \Sigma^{-1} \hat{\mu}$.

We also set the minimum compensation level at $b_{0}=1 \%$. Fig. 2 shows the optimal factor $b$ for two levels of ambiguity aversion of the investor, corresponding to confidence levels $p=10 \%$ and $p=70 \%$ corresponding, respectively, to $\delta=0.078$ and $\delta=0.267$, obtained as quantiles of the distribution (14). The investor uses the incentive to increase the risk aversion of the manager when needed, that is when the Sharpe ratio of the conventional strategy $\hat{H}$ falls below the level $\delta$ of ambiguity aversion. For this reason $b^{*}$ is increasing with the level of ambiguity aversion. For lower levels of $\delta$, like those corresponding to the confidence $p=10 \%$, there is hardly ever any necessity of increasing the fee over the minimum requested by the manager. When $\hat{H}$ is lower than $\delta, b^{*}$ is above $b_{0}$ and reaches its maximum for $\hat{H}$ equal to $\delta / 2$. The times of the occurrences of the peaks observed in Fig. 2 correspond to the months where $\hat{H}$ approaches the level $\delta / 2$ (see Fig. 1), while the peaks' heights depend on the assumption on the term $\alpha W_{0}$ given by (15).

In Fig. 3 we study the effects of the restrictions of the investment set on managerial compensations, with or without ambiguity, where we computed the minimal required sharing factor for the different cases analyzed in Section 4. We consider as the alternative investment set always the total universe of assets and represent the differences between $b_{0}$ and $\bar{b}_{0}$ in the following cases

1. Case 1. The investment is restricted to the green investment set. No ambiguity aversion.

2. Case 2. The investment is restricted to the black investment set. No ambiguity aversion.

3. Case 3. The investment is restricted to the green investment set. With ambiguity aversion at level $\epsilon_{2}=0.267$ for the total universe and $\epsilon_{1}=0.175$ for the restricted set (corresponding to a confidence level $p=0.70$ ).

4. Case 4 . The investment is restricted to the black investment set. With ambiguity aversion at level $\epsilon_{2}=0.267$ for the total universe and $\epsilon_{1}=0.175$ for the restricted set.

The manager's risk aversion is assumed to satisfy (15). We compare cases 1-3 and 2-4 if we want to observe the effect of ambiguity, while we compare cases 1-2 and 3-4 when we want to observe the differences between green and black strategy and the effect of restriction. We note that the premium for cases 1 and 2 are always greater than or equal to zero, as expected, since they represent the compensation for the restriction in the investment set without ambiguity. The compensation for restricting to green assets (case 1) is usually greater, because of the higher expected Sharpe ratio of the black strategy, documented in Fig. 1. When ambiguity aversion is taken into account (cases 3 and 4), the premium becomes smaller and sometimes it gets negative. Negative values mean that an

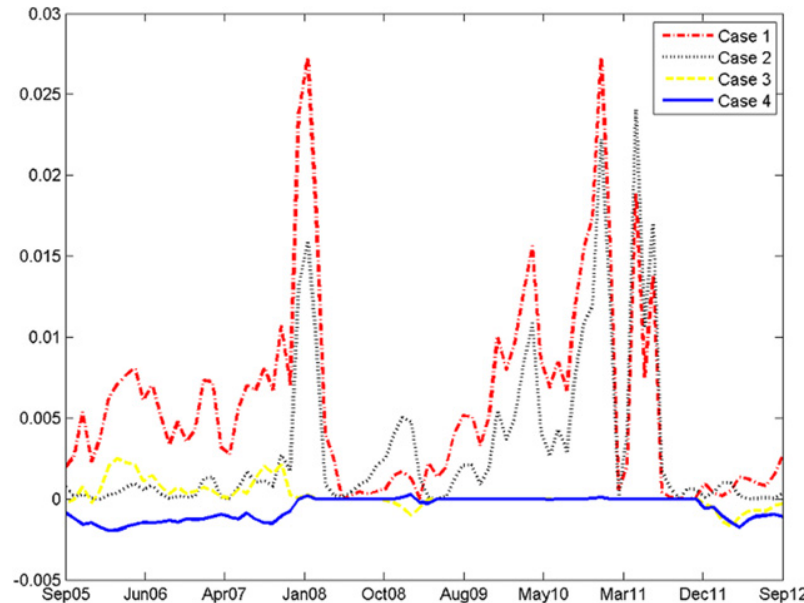

Fig. 3. Changes in the minimum fee required for restricting the investment set to set $X$, with or without ambiguity aversion. Case $1: X$ is the green set, no ambiguity aversion. Case 2: $X$ is the black set, no ambiguity aversion. Case $3: X$ is the green set, with ambiguity aversion. Case $4: X$ is the black set, with ambiguity aversion. The level of ambiguity aversion is obtained by setting a likelihood $p=70 \%$ and the manager's risk aversion from (15).

ambiguity averse manager prefers to invest in the restricted set rather than in the larger one. This happens when the level of ambiguity aversion is high with respect to the expected Sharpe Ratio. Hence, a negative value represents a reduction that the manager is willing to apply on the sharing factor. For a long period starting in January 2008 and going as far as September 2012, the values are zeros or negative for both the black and the green strategies, therefore an ambiguity averse manager would have not asked for any extra compensation for restricting the investment set. The cases 1 , 3 and 2, 4 involve the same kind of restriction, with or without Ambiguity Aversion. Hence, by comparing 1-3 and 2-4 we can isolate the effect of manager's Ambiguity Aversion, observing that it has the effect of diminishing his requests.

In summary, we observed that the aversion to ambiguity on estimated expected returns has a negative effect on the expected Sharpe Ratio of a strategy and, as shown by Fig. 3, decreases the compensation required by a manager to restrict the investment set. For most of the period examined the black strategy had a better Sharpe ratio, therefore the required compensation for managers investing in it were generally lower than the one for the green strategy. However, if a risk-neutral investor believes that expected returns of green assets are easier to predict because of a more transparent governance, as claimed by some advocates of Corporate Social Responsibility (see e.g. [1]), this would affect her own level of ambiguity aversion and, as shown by Fig. 2, decrease the compensation factor for the portfolio manager.

\section{Acknowledgments}

This study was done within the MISTRA programme "Sustainable Investment", funded by the Foundation for Strategic Environmental Research, MISTRA. Comments of an anonymous referee are gratefully acknowledged.

\section{References}

[1] L. Becchetti, R. Ciciretti, A. Giovannelli, Corporate social responibility and earnings forecasting unbiasedness, J. Bank. Finance 37 (9) (2013) 3654-3668.

[2] A. Ben-Tal, A. Nemirovski, Robust convex optimization, Math. Oper. Res. 23 (4) (1998) 769-805.

[3] A. Ben-Tal, A. Nemirovski, Robust solutions to uncertain linear programming problems, Oper. Res. Lett. 25 (1) (1999) 1-13.

[4] D. Bertsimas, D. Pachamanova, M. Sim, Robust linear optimization under general norms, Oper. Res. Lett. 32 (6) (2004) 510-516.

[5] M. Best, R. Grauer, Sensitivity analysis for mean-variance portfolio problems, Manag. Sci. 37 (8) (1991) 980-989. 
[6] M. Best, R. Grauer, On the sensitivity of mean-variance-efficient portfolios to changes in asset means: some analytical and computational results, Rev. Financ. Stud. 4 (2) (1991) 315-342.

[7] S. Bhattacharya, P. Pfleiderer, Delegated portfolio management, J. Econom. Theory 36 (1985) 1-25.

[8] F. Black, R. Litterman, Global portfolio optimization, Financ. Anal. J. 48 (5) (1992) 28-43.

[9] S. Ceria, R. Stubbs, Incorporating estimation errors into portfolio selection: robust portfolio construction, J. Asset Manag. 7 (2) (2006) 109-127.

[10] L. Chen, S. He, S. Zhang, Tight bounds for some risk measures, with applications to robust portfolio selection, Oper. Res. 59 (4) (2011) 847-865.

[11] E. Delage, Y. Ye, Distributionally robust optimization under moment uncertainty with application to data-driven problems, Oper. Res. 58 (3) (2010) 596-612.

[12] V. DeMiguel, F. Nogales, Portfolio selection with robust estimation, Oper. Res. 57 (3) (2009) 560-577.

[13] F. Fabozzi, D. Huang, G. Zhou, Robust portfolios: contributions from operations research and finance, Ann. Oper. Res. 176 (2010) 191-220.

[14] F. Fabozzi, P. Kolm, D. Pachamanova, S. Focardi, Robust portfolio optimization, J. Portfolio Manag. 33 (2007) 40-48.
[15] A. Fabretti, S. Herzel, Delegated portfolio management with socially responsible investment constraints, Eur. J. Finance 18 (3-4) (2012) 293-309.

[16] A. Fabretti, S. Herzel, M. Pinar, Delegated Portfolio Management Under Ambiguity Aversion, Tech. Rep., University of Rome II, Rome, Italy, 2014.

[17] L. Garlappi, R. Uppal, T. Wang, Portfolio selection with parameter and model uncertainty: a multi-prior approach, Rev. Financ. Stud. 20 (1) (2007) 41-81.

[18] L.E. Ghaoui, M. Oks, F. Oustry, Worst-case value-at-risk and robust portfolio optimization: a conic optimization approach, Oper. Res. 51 (4) (2003) 543-556.

[19] D. Goldfarb, G. Iyengar, Robust portfolio selection problems, Math. Oper. Res. 28 (1) (2003) 1-38.

[20] R.A. Johnson, D.W. Wichern, Applied Multivariate Statistical Analysis, fifth ed. Prentice Hall, New Jersey, 1997.

[21] M. Pınar, R. Tütüncü, Robust profit opportunities in risky financial portfolios, Oper. Res. Lett. 33 (2005) 331-340.

[22] L. Stracca, Delegated portfolio management: a survey of the theoretical literature, J. Econom. Surveys 20 (5) (2006) 823-848.

[23] R. Tütüncü, M. Koenig, Robust asset allocation, Ann. Oper. Res. 132 (1) (2004) 157-187. 